13 Rughani J, Deane FP, Wilson CJ. Rural adolescents' help-seeking intentions for emotional problems: the influence of perceived benefits and stoicism. Aust J Rural Health 2011; 19: 64-9.

14 Kataoka S, Zhang L, Wells K. Unmet need for mental health care among US children: variation by ethnicity and insurance status. Am J Psychiatry 2002; 159: 1548-55.

15 Scott LD, Munson MR, Mcmillan JC, Snowden LR. Predisposition to seek mental health care among black male transitioning from foster care. Child Youth Serv Review 2007; 29: 870-82.

16 Chandra A, Minkovitz CS. Stigma starts early: gender differences in teen willingness to use mental health services. J Adoles Health 2006; 38: 754e1-8.

17 Tamres LK, Janicki D, Helgeson VS. Sex differences in coping behaviour: a meta-analytic review and an examination of relative coping. Pers Soc Psychol Rev 2002; 6: 2-29.

18 Sheikh S, Furnham A. A cross-cultural study of mental health beliefs and attitudes towards seeking professional help. Soc Psychiatry Psychiatr Epidemiol 2000; 35: 326-34.

19 Fox C, Buchanan-Barrow E, Barrett M. Children's understanding of mental illness: an exploratory study. Child Care Health Dev 2008; 34: 10-18.

20 Bailey S. Young people, mental illness, and stigmatization. Psychiatr Bull 1999; 23:107-10.
21 Maas E, Marecek J, Travers JR. Children's conceptions of disordered behavior. Child Dev 1978; 49: 146-54.

22 Jones EE, Nisbett RE. The Actor and the Observer: Divergent Perceptions of the Causes of Behavior. General Learning Press, 1971.

23 Martin JK, Pescosolido BA, Olafsdottir S, Mcleod JD. The construction of fear: Americans' preferences for social distance from children and adolescents with mental health problems. J Health Soc Behav 2007; 48: 50-67.

24 Phelan JC. Geneticization of deviant behavior and consequences for stigma: the case of mental illness. J Health Soc Behav 2005; 46: 307-22.

25 Dietrich S, Beck M, Bujantugs B, Kenzine D, Matschinger $\mathrm{H}$, Angermeyer MC. The relationship between public causal beliefs and social distance toward mentally ill people. Aust N Z J Psychiatry 2004; 28: 348-54.

26 Corrigan PW, Lurie BD, Goldman HH, Slopen N, Medasani K, Phelan S. How adolescents perceive the stigma of mental illness and alcohol abuse. Psychiatr Serv 2005; 56: 544-50.

27 Martin JK, Pescosolido BA, Tuch SA. Of fear and loathing: the role of 'disturbing behavior' labels, and causal attributions in shaping public attitudes towards people with mental illness. J Health Soc Behav 2000; 41: 208-23.

28 McGoldrick M, Giordano J, Pearce JK. Ethnicity and Family Therapy. Guilford, 1996.

\title{
Trauma exposure and refugee status as predictors of mental health outcomes in treatment-seeking refugees
}

Jeroen W. Knipscheer, ${ }^{1,2}$ Marieke Sleijpen, ${ }^{1,2}$ Trudy Mooren, ${ }^{1}$ F. Jackie June ter Heide, ${ }^{1}$ Niels van der $A a^{1}$

BJPsych Bulletin (2015), 39, 178-182, doi: 10.1192/pb.bp.114.04795

${ }^{1}$ Arq Psychotrauma Expert Group, Diemen/Oegstgeest, The Netherlands: ${ }^{2}$ Utrecht University, Department of Clinical and Health Psychology, Utrecht, The Netherlands

Correspondence to Jeroen W. Knipscheer (j.w.knipscheer@uu.nl)

First received 30 Apr 2014, final revision 14 Nov 2014, accepted 4 Dec 2014

(C) 2015 The Authors. This is an open-access article published by the Royal College of Psychiatrists and distributed under the terms of the Creative Commons Attribution License (http://creativecommons.org/ licenses/by/4.0), which permits unrestricted use, distribution, and reproduction in any medium, provided the original work is properly cited.
Aims and method This study aimed to identify predictors of symptom severity for post-traumatic stress disorder (PTSD) and depression in asylum seekers and refugees referred to a specialised mental health centre. Trauma exposure (number and domain of event), refugee status and severity of PTSD and depression were assessed in 688 refugees.

Results Symptom severity of PTSD and depression was significantly associated with lack of refugee status and accumulation of traumatic events. Four domains of traumatic events (human rights abuse, lack of necessities, traumatic loss, and separation from others) were not uniquely associated with symptom severity. All factors taken together explained $11 \%$ of variance in PTSD and depression.

Clinical implications To account for multiple predictors of symptom severity including multiple traumatic events, treatment for traumatised refugees may need to be multimodal and enable the processing of multiple traumatic memories within a reasonable time-frame.

Declaration of interest None.
Refugees and asylum seekers have been shown to be at substantially higher risk of developing post-traumatic stress disorder (PTSD) ${ }^{1}$ and comorbid mental health problems than the general population, ${ }^{2}$ compatriots who have stayed in the refugees' home country, ${ }^{3}$ and economic migrants. ${ }^{4}$ Exposure to a high number of potentially traumatic events, involvement in asylum-seeking procedures and forced migration are stressors that set refugees apart from other 
populations and increase their psychological vulnerability. In Western psychiatric practice, insight in the determinants of PTSD and comorbid disorders in refugees may be helpful in guiding treatment interventions. Meta-analyses have shown that torture and cumulative traumatic events are the main predictors for development of PTSD in refugees. ${ }^{5}$ Additionally, researchers have begun to investigate whether different symptom profiles in refugees are related to different traumatic experiences. In a sample of Bosnian refugees, Momartin et $a l^{6}$ found that life threat and traumatic loss were related to PTSD and depression. In a sample of Mandean refugees, Nickerson et $a l^{7}$ found that PTSD and prolonged grief disorder were predicted by traumatic loss, whereas PTSD only was predicted by difficulties related to loss of culture and support.

While these findings are highly relevant to treatmentseeking refugees, similar analyses have not yet been carried out in large, treatment-seeking refugee samples. In this study we examine whether symptom severity of PTSD and depression is predicted by refugee status as well as accumulation and specific domains of traumatic events in a sample of refugees seeking specialised mental healthcare.

\section{Method}

\section{Participants}

Participants were asylum seekers and refugees referred for treatment at Foundation Centrum '45, a specialised Dutch centre for diagnosis and treatment of complex psychological trauma. Refugees are individuals who have been granted temporary or permanent refugee status in The Netherlands whereas asylum seekers are those still awaiting a final decision. In 2001, Foundation Centrum '45 started to routinely monitor treatment outcomes by administering questionnaires to patients at intake and annually during treatment. For the present study, data collected at intake were used. Complete data with regard to refugee status, traumatic experiences and symptom severity of PTSD and depression were available for 688 asylum seekers and refugees. Characteristics for the total sample are presented in Table 1. Participants came from three main regions: 58\% from the Middle East (e.g. Afghanistan, Iraq, Egypt, Libya), 17\% from Sub-Saharan Africa (e.g. Burkina Faso, Eritrea, Congo) and $16 \%$ from Balkan Europe (Bosnia, former Yugoslavia, Croatia).

\section{Measures}

Traumatic experiences and PTSD symptom severity were assessed using the Harvard Trauma Questionnaire (HTQ). ${ }^{8}$ The HTQ consists of three parts, the first two of which were used in this study. In the first part participants were asked to indicate which of the 19 traumatic events they experienced, witnessed or heard of, and only self-experienced traumatic events were used in the study. For each participant a total number of different self-experienced traumatic events was calculated (range 0-19). In the second part of the HTQ the severity of DSM-IV PTSD symptoms was assessed by asking participants how much they were bothered by 16 PTSD symptoms during the past week, rated on a 4-point scale ranging from 1 , not at all to 4 , extremely.
PTSD symptom severity was computed by averaging responses (range 1-4). The HTQ recommends a cut-off score of 2.5 to identify clinically significant PTSD. Internal consistency of the scale was high (Cronbach's $\alpha 0.86$ ).

Symptom severity for depression was assessed with the Hopkins Symptom Checklist (HSCL-25). ${ }^{9}$ Participants were asked to indicate how distressed they were by rating 10 symptoms of anxiety and 15 symptoms of depression during the past week on a 4-point scale ranging from 1, not at all to 4, extremely. Symptom severity of depression (range 1-4) was computed by averaging responses on the 15 depression items. The HSCL-25 recommends a cut-off score of 1.75 to indicate clinically significant depression. Internal consistency was high (Cronbach's $\alpha$ 0.89).

The HTQ and HSCL-25 are self-report questionnaires that are widely used with refugees and are available in many different languages. Questionnaires were administered in the patient's native language if possible and interpreters were used when necessary. Both instruments have good psychometric properties. ${ }^{10}$

\section{Statistical analyses}

Statistical analyses were performed using SPSS version 20. To investigate whether specific domains of traumatic events could be identified, a principal component analysis with oblique rotation (direct oblimin) was conducted on the total set of self-experienced traumatic events. An initial analysis was run to obtain eigenvalues for each factor in the data and to evaluate the substantive contribution of each item to the extracted factors. Stevens ${ }^{11}$ recommends interpreting factor loadings greater than 0.4 as substantive. The analysis was rerun without the items that did not contribute substantively to the extracted factors. For each participant a total score was computed on each of the extracted domains of traumatic events by counting the total number of self-experienced traumatic events within the domain.

Hierarchical regression analyses were used to test whether refugee status, total number of self-experienced traumatic events and the domains of traumatic events predicted symptom severity of PTSD and depression. These variables were independently added to the regression models, together with the gender and age covariates, as these have been found to predict PTSD in refugee samples. ${ }^{12}$ Scores on the extracted domains of traumatic events were recoded into dummy variables before being added to the hierarchical regression models. To allow for multiple tests the alpha level of significance was set to 0.01 .

\section{Results}

First, it was investigated whether specific domains of traumatic events could be identified by conducting a principal component analysis. In the initial analysis, four factors had eigenvalues greater than 1. Four traumatic events (combat situation, brainwashing, rape or sexual abuse, and being close to death) did not contribute substantively to any of the four extracted factors and the analysis was rerun without these items. Four factors were retained with eigenvalues greater than 1.0, which together accounted for $56.7 \%$ of the total variance. Table 2 presents

\section{Bulletin}


Table 1 Demographic characteristics of the total sample and the main regions of origin

\begin{tabular}{lccccc} 
& $\begin{array}{c}\text { Total sample } \\
(n=688)\end{array}$ & $\begin{array}{c}\text { Middle East } \\
(n=397)\end{array}$ & $\begin{array}{c}\text { Sub-Saharan Africa } \\
(n=114)\end{array}$ & $\begin{array}{c}\text { Balkan Europe } \\
(n=111)\end{array}$ & $\begin{array}{c}\text { Other regions } \\
(n=66)\end{array}$ \\
\hline Male, $n$ (\%) & $488(71)$ & $306(77)$ & $66(58)$ & $70(63)$ & $45(68)$ \\
\hline Age, mean (s.d.) & $40.4(10.4)$ & $41.3(9.9)$ & $32.2(8.5)$ & $45.2(9.1)$ & $41.4(11.2)$ \\
\hline Permanent refugee status, $n$ (\%) & $585(85)$ & $361(91)$ & $73(64)$ & $107(96)$ & $44(67)$ \\
\hline
\end{tabular}

\begin{tabular}{|c|c|c|c|c|}
\hline & \multicolumn{4}{|c|}{ Rotated factor loadings } \\
\hline & $\begin{array}{l}\text { Human rights } \\
\text { abuses }\end{array}$ & $\begin{array}{l}\text { Traumatic } \\
\text { loss }\end{array}$ & $\begin{array}{l}\text { Lack of basic } \\
\text { human needs }\end{array}$ & $\begin{array}{l}\text { Separation } \\
\text { from others }\end{array}$ \\
\hline Threatened to be executed & 0.78 & 0.13 & -0.13 & 0.09 \\
\hline Physical torture & 0.77 & -0.10 & -0.03 & -0.12 \\
\hline Threatened to be physically tortured & 0.77 & -0.03 & -0.10 & -0.08 \\
\hline Threatened to watch torturing & 0.66 & 0.04 & 0.07 & -0.04 \\
\hline Serious injury & 0.50 & 0.01 & 0.21 & 0.11 \\
\hline Lost or kidnapped & 0.46 & 0.15 & 0.13 & -0.04 \\
\hline Imprisonment & 0.46 & -0.04 & 0.16 & -0.28 \\
\hline Murder of family or friend & 0.02 & 0.85 & -0.04 & 0.01 \\
\hline Unnatural death of family or friend & -0.12 & 0.79 & 0.04 & -0.11 \\
\hline Murder of stranger or strangers & 0.15 & 0.63 & 0.03 & 0.02 \\
\hline Lack of shelter & -0.03 & 0.05 & 0.79 & 0.12 \\
\hline Lack of food or water & -0.07 & 0.03 & 0.78 & -0.15 \\
\hline III health without access to medical care & 0.13 & -0.03 & 0.68 & -0.07 \\
\hline Forced separation from family members & -0.04 & 0.09 & -0.02 & -0.85 \\
\hline Forced isolation from others & 0.14 & 0.03 & 0.06 & -0.75 \\
\hline
\end{tabular}

a. Factor loadings over 0.40 appear in bold.

the factor loadings after rotation. The traumatic events that cluster on the same factor suggest that the first factor represents human rights abuses $(31.1 \%$ of the total variation), the second factor traumatic loss ( $10.0 \%$ of the total variation), the third factor a lack of necessities (8.7\% of the total variation), and the fourth factor separation from others $(6.9 \%$ of the total variation).

Mean symptom severity was 3.1 (s.d. 0.5) for PTSD and 2.9 (s.d. 0.6) for depression both within the clinical range. A clinical level of symptom severity for PTSD and depression was reported by, respectively, $84 \%$ and $95 \%$ of participants. Participants reported a mean of 11.2 different self-experienced traumatic events (s.d. 4.7). The most commonly reported events were being close to death (80\%), forced separation from family members (74\%), murder of family or friend $(72 \%)$, threatened to be physically tortured (72\%), and unnatural death of family or friend $(66 \%)$. With regard to the trauma domains, human rights abuses were reported by $90 \%$ of participants, traumatic losses by $83 \%$, lack of necessities by $77 \%$, and separation from others by $81 \%$.

Hierarchical regression analyses were used to test whether possession of refugee status, the total number of different self-experienced traumatic events, and different domains of self-experienced traumatic events predicted symptom severity of PTSD and depression. Results of the hierarchical regression models are shown in Table 3. First, symptom severity of PTSD and depression was adjusted for gender and age by adding them to the model in step 1 . Refugee status was added to the model in step 2. Lack of refugee status was significantly associated with increased symptom severity for PTSD and depression. Refugee status accounted for $2 \%$ of the variation in symptom severity of those disorders. Total number of different self-experienced traumatic events was added to the model in step 3. Increased number of different self-experienced traumatic events was significantly associated with increased symptom severity for PTSD and depression. Total number of different self-experienced traumatic events accounted for $8 \%$ of the variation in PTSD symptom severity and for $7 \%$ of the variation in symptom severity for depression. To test the unique effect of different domains of self-experienced traumatic events to symptom severity of PTSD and depression, human rights abuses, traumatic loss, lack of necessities and separation from others were added to the model in step 4 . None of these domains were significantly associated with symptom severity. Adding the different domains of self-experienced traumatic events to the model accounted for an additional $1 \%$ of the variation in symptom severity. 
Table 3 Hierarchical regression models of predictors of symptom severity with regard to PTSD and depression ${ }^{\text {a }}$

\begin{tabular}{|c|c|c|c|c|c|c|c|c|}
\hline & \multicolumn{4}{|c|}{ PTSD symptoms } & \multicolumn{4}{|c|}{ Depressive symptoms } \\
\hline & B & s.e. & Beta & $\Delta R^{2}$ & B & s.e. & Beta & $\Delta R^{2}$ \\
\hline Step 1 & & & & 0.00 & & & & 0.01 \\
\hline Constant & 3.05 & 0.03 & & & 2.88 & 0.03 & & \\
\hline Gender & 0.04 & 0.05 & 0.03 & & 0.11 & 0.05 & 0.08 & \\
\hline Age & 0.02 & 0.02 & 0.03 & & 0.03 & 0.02 & 0.05 & \\
\hline Step 2 & & & & $0.02 *$ & & & & $0.02 *$ \\
\hline Constant & 3.02 & 0.03 & & & 2.84 & 0.03 & & \\
\hline Gender & 0.04 & 0.05 & 0.04 & & 0.12 & 0.05 & 0.09 & \\
\hline Age & 0.04 & 0.02 & 0.07 & & 0.06 & 0.02 & 0.09 & \\
\hline Refugee status & 0.21 & 0.06 & $0.14^{*}$ & & 0.28 & 0.07 & $0.16^{*}$ & \\
\hline Step 3 & & & & $0.08^{*}$ & & & & $0.07^{*}$ \\
\hline Constant & 3.00 & 0.03 & & & 2.82 & 0.03 & & \\
\hline Gender & 0.09 & 0.04 & 0.08 & & 0.18 & 0.05 & $0.13^{*}$ & \\
\hline Age & 0.04 & 0.02 & 0.07 & & 0.06 & 0.02 & 0.09 & \\
\hline Refugee status & 0.20 & 0.06 & $0.13^{*}$ & & 0.26 & 0.07 & $0.15^{*}$ & \\
\hline Traumatic experiences, $n$ & 0.15 & 0.02 & $0.28^{*}$ & & 0.17 & 0.02 & $0.28^{*}$ & \\
\hline Step 4 & & & & 0.01 & & & & 0.01 \\
\hline Constant & 2.82 & 0.11 & & & 2.58 & 0.13 & & \\
\hline Gender & 0.09 & 0.04 & 0.08 & & 0.17 & 0.05 & $0.12^{*}$ & \\
\hline Age & 0.04 & 0.02 & 0.07 & & 0.06 & 0.02 & 0.09 & \\
\hline Refugee status & 0.20 & 0.06 & $0.13^{*}$ & & 0.26 & 0.07 & $0.15^{*}$ & \\
\hline Traumatic experiences, $n$ & 0.11 & 0.03 & 0.21 * & & 0.12 & 0.04 & $0.18^{*}$ & \\
\hline Human right abuses & 0.15 & 0.08 & 0.09 & & 0.09 & 0.09 & 0.05 & \\
\hline Traumatic loss & 0.01 & 0.06 & 0.00 & & 0.07 & 0.07 & 0.04 & \\
\hline Lack of basic human needs & 0.05 & 0.06 & 0.04 & & 0.04 & 0.07 & 0.03 & \\
\hline Separation from others & 0.01 & 0.06 & 0.01 & & 0.09 & 0.07 & 0.06 & \\
\hline
\end{tabular}

B, Unstandardised regression coefficient; Beta, standardised regression coefficient; PTSD, post-traumatic stress disorder. a. Dependent variables: symptom severity with regard to PTSD and depression. ${ }^{\star} P<0.01$

$\Delta R^{2}$, change in $R^{2}$ compared with previous step.

\section{Clinical implications}

In a large sample of asylum-seeking and refugee patients seeking treatment within a specialised Western mental health setting, PTSD symptom severity and depression was predicted by lack of refugee status and cumulative traumatic events, but not by specific domains of traumatic experience. Refugee status, total number of self-experienced traumatic events, domains of traumatic experiences, and gender and age together accounted for only $11 \%$ of variation in symptom severity of PTSD and depression. These results are in stark contrast with earlier findings among nontreatment-seeking refugee populations, which showed that torture and cumulative traumatic events accounted for $34.4 \%$ of variance in PTSD prevalence rates and for $33.4 \%$ of variance in depression prevalence rates. ${ }^{5}$

Clearly, PTSD symptom and depression severity among asylum seekers and refugees seeking specialised treatment is influenced by multiple factors, including some that were not measured in this study. To map predictors for PTSD and depression in refugee patients, assessment may need to focus on a broader range of both stressors and resources, including stressors and resources related to forced migration, such as safety of family in the home country and social support. Rather than traumatic stress, it may be the burden of current stress and lack of resources that leads to PTSD and depression, prompting refugees to seek mental healthcare. This finding implies that clinically, in this severely traumatised population, an exclusive focus on processing of traumatic experiences as prescribed in PTSD treatment guidelines may result in only limited symptom reduction. Consequently, for refugee patients with severe psychopathology treatment may need to be multimodal rather than trauma-focused only.

In addition, in contrast to other studies, which showed an association between life threat and traumatic loss on the one hand and PTSD and comorbid disorders on the other, in our treatment-seeking sample no such associations were found. In our sample, the number rather than domain of traumatic events was associated with symptom severity. This implies that trauma-focused treatments for refugees should be designed to enable the processing of a large number of traumatic events within a reasonable time-frame. Treatments such as narrative exposure therapy and traumafocused cognitive therapy may enable that to a greater extent than in vitro exposure therapy or eye movement desensitisation and reprocessing therapy, which in refugees may require several sessions for the processing of a single memory.

Although our findings can be generalised to mental healthcare-seeking refugees and asylum seekers only and the range of questionnaires was limited, merits of this study lie in the satisfactory cultural validity of the questionnaires and the large sample size. Future studies using a broader range of instruments are needed to identify predictors for PTSD and depression in treatment-seeking refugees.

\section{About the authors}

Jeroen W. Knipscheer is a psychologist and senior researcher and Marieke Sleijpen is a psychologist and PhD student, both at Arq Psychotrauma 
Expert Group, Diemen/Oegstgeest, The Netherlands and Department of Clinical and Health Psychology, Utrecht University; Trudy Mooren is a clinical psychologist and senior researcher, F. Jackie June ter Heide is a clinical psychologist and $\mathrm{PhD}$ student and Niels van der $\mathbf{A a}$ is a senior researcher and methodologist, all with Arq Psychotrauma Expert Group, Diemen/Oegstgeest.

\section{References}

1 American Psychiatric Association. Diagnostic and Statistical Manual of Mental Disorders, Fourth Edition, Text Revision (DSM-IV-TR). APA, 2000.

2 Fazel $M$, Jeremy $W$, Danesh J. Prevalence of serious mental disorder in 7000 refugees resettled in western countries: a systematic review. Lancet 2005; 365: 130914

3 Porter M, Haslam N. Predisplacement and postdisplacement factors associated with mental health of refugees and internally displaced persons: a meta-analysis. JAMA 2005; 294: 60212.

4 Lindert J, von Ehrenstein OSV, Priebe S, Mielck A, Brähler E. Depression and anxiety in labor migrants and refugees a systematic review and meta-analysis. Soc Sci Med 2009; 69: 24657.

5 Steel Z, Chey T, Silove D, Marnane C, Bryant RA, Van Ommeren M Association of torture and other potentially traumatic events with mental health outcomes among populations exposed to mass conflict and displacement: a systematic review and meta-analysis. JAMA 2009; 302: 53749.
6 Momartin S, Silove D, Manicavasagar V, Steel Z. Comorbidity of PTSD and depression: associations with trauma exposure, symptom severity and functional impairment in Bosnian refugees resettled in Australia. J Affect Disord 2004; 80: 2318.

7 Nickerson A, Liddell BJ, Maccallum F, Steel Z, Silove D, Bryant RA. Posttraumatic stress disorder and prolonged grief in refugees exposed to trauma and loss. BMC Psychiatry 2014; 14: 106.

8 Mollica RF, Caspi-Yavin Y, Bollini P, Truong T, Tor S, Lavelle J. The Harvard Trauma Questionnaire: validating a cross-cultural instrument for measuring torture, trauma, and posttraumatic stress disorder in Indochinese refugees. J Nerv Ment Dis 1992; 180: 1116.

9 Mollica RF, Wyshak G, De Marnette T, Tu B, Yang T, Khuon F, et al. Hopkins Symptom Checklist (HSCL-25): Manual for Cambodian, Laotian and Vietnamese versions. Torture 1996; 6: 3542

10 Hollifield M, Warner TD, Lian N, Krakow B, Jenkins JH, Kesler J, et al Measuring trauma and health status in refugees: a critical review. JAMA 2002; 288: 61121.

11 Stevens JP. Applied Multivariate Statistics for the Social Sciences. Erlbaum, 2002

12 Johnson $\mathrm{H}$, Thompson A. The development and maintenance of post traumatic stress disorder (PTSD) in civilian adult survivors of war trauma and torture: a review. Clin Psychol Rev 2008; 28: 3647.

\title{
Difficult to treat? A comparison of the effectiveness of treatment as usual in refugees and non-refugees
}

\author{
F. Jackie June ter Heide, ${ }^{1}$ Geert E. Smid ${ }^{1}$
}

BJPsych Bulletin (2015), 39, 182-186, doi: 10.1192/pb.bp.114.047928

${ }^{1}$ Foundation Centrum '45/partner in Arq, The Netherlands

Correspondence to Jackie June ter Heide (j.ter.heide@centrum45.nl)

First received 30 Apr 2014, final revision 20 Feb 2015, accepted 12 Mar 2015

(C) 2015 The Authors. This is an openaccess article published by the Royal College of Psychiatrists and distributed under the terms of the Creative Commons Attribution License (http:// creativecommons.org/licenses/by/ 4.0), which permits unrestricted use, distribution, and reproduction in any medium, provided the original work is properly cited.
Aims and method To examine treatment response in traumatised refugees, we compared routine outcome monitoring data (Harvard Trauma Questionnaire) of two refugee populations with those of individuals experiencing profession-related trauma who were treated at a specialised psychotrauma institute.

Results Asylum seekers/temporary refugees $(n=21)$ and resettled refugees $(n=169)$ showed significantly lower post-traumatic stress disorder (PTSD) symptom reduction between intake and 1 year after intake than did a comparison group of non-refugees $(n=37)$, but the interaction effect was clinically small (partial $\eta^{2}=0.03$ ). Refugees who had more severe symptoms at intake showed significantly greater symptom reduction after 1 year.

Clinical implications Therapists and refugee patients should have realistic expectations about response to treatment as usual. Additional treatment focusing on improving quality of life may be needed for refugees whose PTSD symptom severity remains high. At the same time, novel approaches may be developed to boost treatment response in refugee patients with low responsiveness.

Declaration of interest None.
Among many clinicians, traumatised asylum seekers and refugees have a reputation of being difficult to treat. Low treatment response in refugees is often attributed to patient-related factors, ${ }^{1}$ such as trauma history, current stressors and complex psychopathology. Many asylum seekers and refugees have been exposed to multiple, prolonged, interpersonal traumatic events such as war and human trafficking. ${ }^{2}$ In addition, they have to handle the 